\title{
Investigation of the relationship between trochlear morphology and medial patellar cartilage defect using magnetic resonance imaging
}

\author{
Semra Duran, Elif GunAYDin
}

From the Ankara Numune Training and Research Hospital, Ankara, Turkey

The aim of this study was to evaluate trochlear morphology in patients with medial patellar cartilage defects via magnetic resonance imaging (MRI).

Three hundred patients who were diagnosed with grade 2, 3 and 4 medial patellar cartilage defect using MRI according to the International Cartilage Repair Society Classification System and 100 control subjects were evaluated. Trochlear morphology was evaluated based on lateral trochlear inclination (LTI), medial trochlear inclination (MTI), sulcus angle( SA), femoral lateral and medial condyle symmetry, trochlear facet asymmetry, and trochlear width on the axial MR images.

The mean SA was significantly higher in the medial patellar cartilage defect group compared to the control group $(p<.05)$. The LTI and MTI of the cartilage defect group were significantly lower than those of the control group $(p<.05)$. With the decreasing LTI and MTI, there was an increase in medial patellar cartilage loss. LTI $(r=-0.46)$ and MTI $(r=-0.53)$ were moderately correlated with SA. There was no significant differences in femoral lateral and medial condyle symmetry, trochlear facet asymmetry, and trochlear width between groups with and without medial patellar cartilge defect $(p>.05)$.

A flattened medial trochlea is a risk factor for cartilage structural damage of the medial patellofemoral joint, and it plays a role in the development of a defect in the medial patellar cartilage. The medial patellar cartilage defect is associated with the flattened lateral trochlea.

Keywords : chondromalacia patella ; femoral trochlea ; morphology ; magnetic resonance imaging.

No benefits or funds were received in support of this study. None of the authors have a conflict of interest.

\section{INTRODUCTION}

A patellar cartilage defect is a frequent pathology associated with pain and reduced quality of life. These lesions lack the ability to heal on their own and may be precursors to osteoarthritis (1-3). Abnormal patellofemoral alignment (e.g., lateral displacement and tilt) and trochlear morphology (e.g., shallow trochlea) are related to patellofemoral osteoarthritis. Anatomical parameters that have been related to trochlear morphology include the sulcus angle (SA), lateral trochlear inclination (LTI), and trochlear facet asymmetry $(2,4-8)$. Previous studies have suggested that with the decreasing LTI (a flattened lateral trochlea), the patella is more likely to be laterally displaced. Thus, the contact between the patella and lateral femoral condyle increases, leading to cartilage damage in the patellofemoral joint (4-6). However, it remains unclear what characteristics of trochlear morphology affect medial patellofemoral osteoarthritis.

Semra Duran ${ }^{1}, \mathrm{MD}$

Elif Gunaydin², MD

${ }^{1}$ Ankara Numune Training and Research Hospital, Ankara MedicalPark Hospital, Department of Radiology, Ankara, Turkey.

Correspondence : Semra Duran, Ankara Numune Training and Research Hospital, Talatpasa Bulvarı No.5 Altındag/ Ankara, Türkiye. Fax : 903123103030.

E-mail : semraduran91@gmail.com

- 2021, Acta Orthopædica Belgica. 
Magnetic resonance imaging (MRI) has emerged as a reliable and accurate investigating modality for patients with anterior knee pain and suspected chondromalacia patellae due to its noninvasive nature, multiplanar imaging capabilities, and refinements in sequences, which allows detailed soft-tissue and cartilage imaging (5,8-11). Crosssectional imaging modalities are the most sensitive, noninvasive techniques for demonstrating trochlear morphology.

The purpose of this study was to evaluate trochlear morphology in patients with medial patellar cartilage defects using MRI.

\section{MATERIALS AND METHODS}

A total of 380 patients who were diagnosed with a grade 2, 3 or 4 medial patellar cartilage defect according to the International Cartilage Repair Society Classification System based on MRI evaluation between January 2017 and December 2018 were retrospectively evaluated. The exclusion criteria were history of knee surgery or trauma, a previous diagnosis of space-occupying knee lesions, being aged $<35$ or $>55$ years, trochlear dysplasia (SA $>144^{\circ}$, trochlear facet asymmetry $<0.4$, and LTI $\left.<11^{\circ}\right)(12,13)$, patella alta/ baja (Insall Salvati ratio $>1.3$ or $<0.8)$ (14), and MR images being motion-degraded or having insufficient quality to accurately assess joint cartilage. After applying the exclusion criteria, 300 patients were included in the medial patellar cartilage defect group. In addition, a control group was composed of 100 patients with normal cartilage morphology as confirmed by MRI. No ethical committee approval was required owing to the retrospective nature of the study.

The MR images of the patients were acquired on a 1. 5-T unit (Optima, GE Medical System, Wilwaukee, Wisconsin, USA) using extremity coil in the supine position with the knee in full extension. A standardized MRI examination protocol was used, and the following five sequences were performed for each patient.

Sagittal fast spin-echo T1-weighted (repetition time (TR)/echo time (TE): 300-500/5-10 ms, matrix : $288 \times 224$, field of view (FOV) : $18 \times 18$ $\mathrm{cm}$, slice thickness : $3 \mathrm{~mm}$ ] ; sagittal fat-suppressed proton density-weighted (PDW) (TR/TE : 23002800/20-40 ms, matrix : $256 \times 192$, FOV : $18 \times 18$ $\mathrm{cm}$, slice thickness : $3 \mathrm{~mm}$ ); coronal fast spin-echo T1-weighted (TR/TE : 300-500/5-10 ms, matrix : $288 \times 224$, FOV : $20 \times 20 \mathrm{~cm}$, slice thickness : 3 $\mathrm{mm})$; coronal fat-suppressed PDW (TR/TE : 23002800/20-40 ms, matrix : $288 \times 224$, FOV : $20 \times 20$ $\mathrm{cm}$, slice thickness : $3 \mathrm{~mm}$ ); and axial fat-suppressed PDW (TR/TE : 2300-2800/20-40 ms, matrix : $288 \times$ 224, FOV : $18 \times 18 \mathrm{~cm}$, slice thickness : $4 \mathrm{~mm}$ ).

To evaluate the presence and size of patellar cartilage defects and determine trochlear measurements, we used axial fat-suppressed PDW sequences. A medial patellar cartilage defect was considered to be present if there was irregularity on the cartilage surface with a loss of cartilage thickness on at least two consecutive slices. The severity of the cartilage defect was determined using lesion depth in accordance with the International Cartilage Repair Society Classification System (4-6) as follows :

Grade 0 - normal cartilage ( control group ) ( Fig I a).

Grade 1 - chondral softening or blistering with an intact surface (not included in the study) (Fig I b ).

Grade $2-$ loss of $<50 \%$ of cartilage thickness without exposed bone (Fig I c).

Grade 3 - loss of $>50 \%$ of cartilage thickness without exposed bone (Fig I d).

Grade 4 - full-thickness cartilage loss with exposed bone (Fig I e).

The defect group was further divided into subgroups according to this classification, and the results of each group were compared with those of the control group.

We evaluated the morphological features of the trochlea using SA, LTI, medial trochlear inclination (MTI), femoral lateral and medial condyle symmetry, trochlear facet asymmetry, and trochlear width. These measurements were obtained from the axial MR images at the level of the anterior cruciate ligament femoral insertion along the osseous surface.

SA was defined as the angle between the medial and lateral trochlear facets (Fig. II a) $(5,6,8)$.

A posterior condylar line was drawn along the most posterior surface of the femoral condyles. 

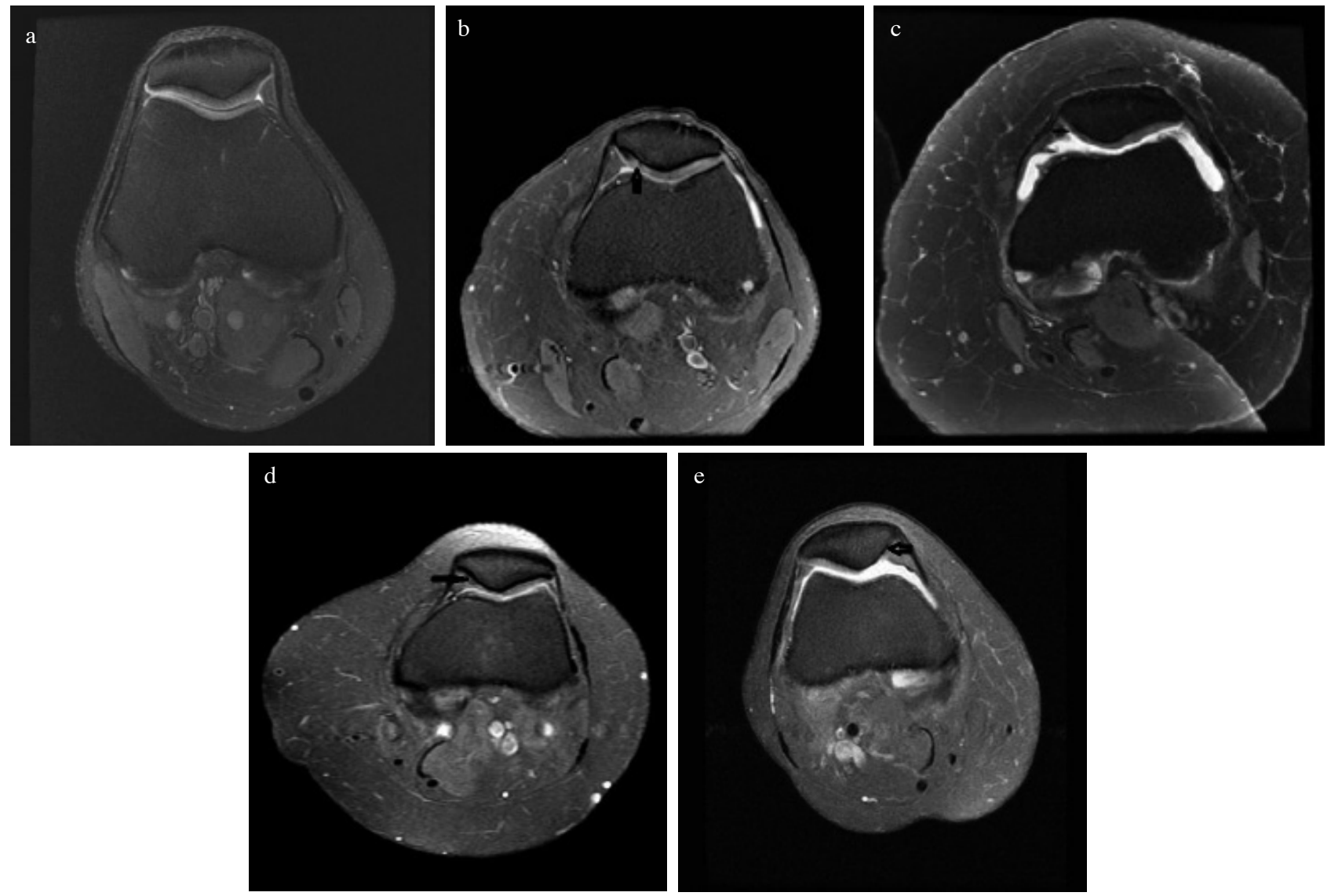

Fig. I. - Axial proton-density fat-suppressed MR images of the knee showing (a) Grade 0 (b) Grade 1 (c) Grade 2 (d) Grade 3 and (e) Grade 4 cartilage defect in the medial facet of the patella (arrows).

LTI was defined as the angle between the posterior condylar line and the line drawn along the surface of the lateral trochlear facet (Fig II b) (4-6).

MTI was defined as the angle between the posterior condylar line and the line drawn along the surface of the medial trochlear facet (Fig II c) $(5,6)$.

Femoral lateral and medial condyle symmetry was defined as the ratio of lateral condyle height to medial condyle height (Fig II d).

Trochlear facet asymmetry was defined by the ratio of medial facet to lateral facet length (Fig II e) $(15,16)$.

Trochlear width was defined as the distance from the line connecting the most anterior parts of the medial and lateral trochlear facets (Fig II f) $(15,16)$.

SPSS (version 20.0 ; SPSS Inc., Chicago, IL) was used for statistical analysis. The distribution between the groups was found to be normal with the Kolmogorov-Smirnov test. Non-homogeneity of data distribution was determined using Levene's test, and parametric tests were used for inter-group comparisons.

We also used paired samples to determine the differences between the groups for continuous data. A t-test for independent samples was used to compare categorical data with two states. Oneway analysis of variance and Bonferroni test were used to compare categorical data with more than two states. To evaluate the relationship between the variables of the patellar cartilage defect group, the Pearson correlation test was performed. The level of statistical significance was considered as .05 .

All measurements of trochlear morphology were repeated at three-week intervals by the first author (S.D). The second author (EG) who was blinded to all subject information performed all measurements 

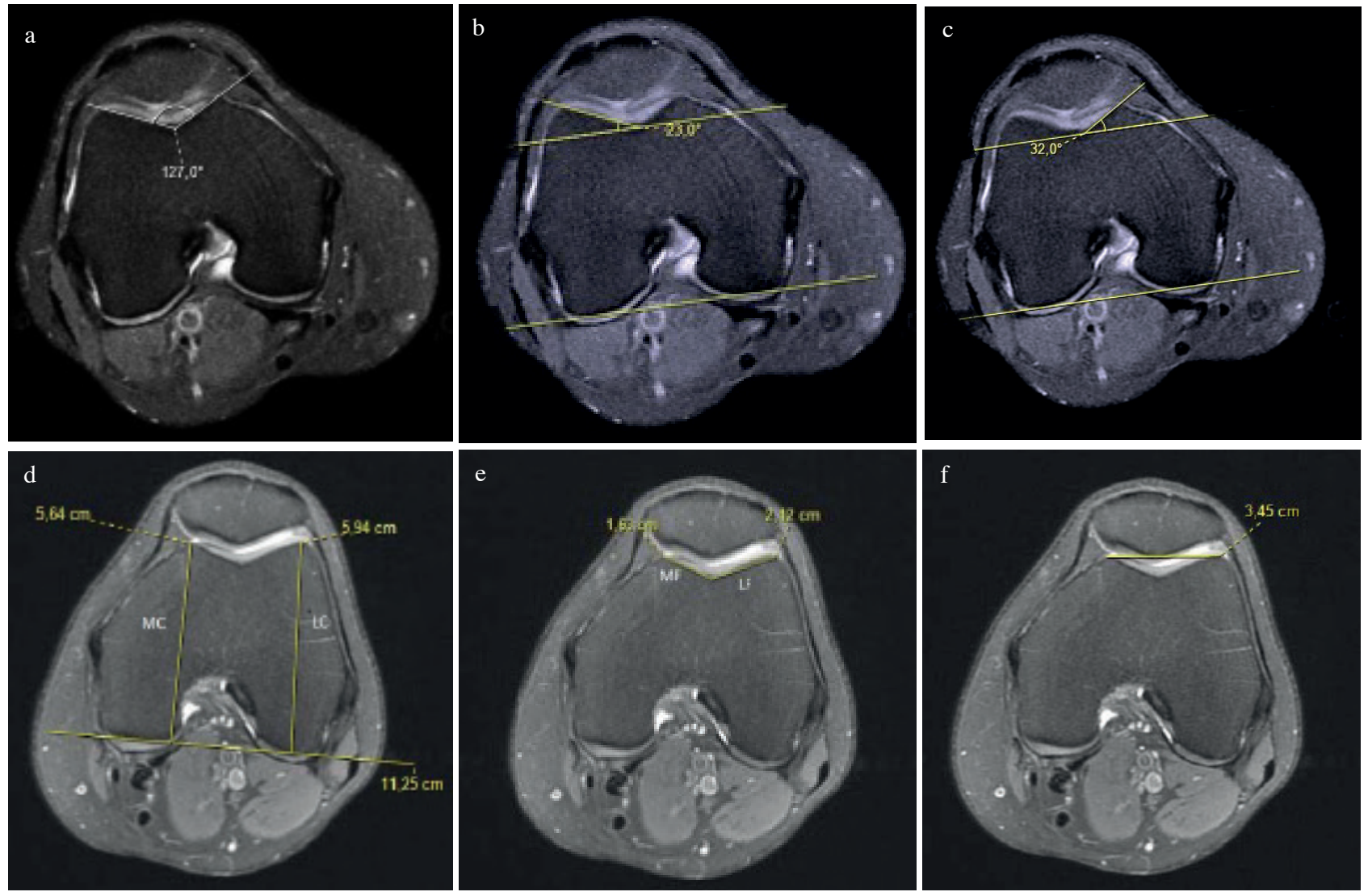

Fig. II. - Measurement of sulcus angle (a), lateral trochlear inclination (b), medial trochlear inclination (c), femoral lateral and medial condyle symmetry (MC : medial condyle, LC : lateral condyle (d), trochlear facet asymmetry (MF : medial facet, LF : lateral facet) (e), and trochlear width (f) in patient on axial proton-density fat-suppressed MR images.

in 150 knees randomly selected from 400 knees. The inter- and-intraobserver intraclass correlation coefficients for all measurements were calculated as 0.86 and 0.88 , respectively.

The characteristics of the study subjects are presented in Table I.

In all defect grades, SA was significantly greater compared to the control group $(\mathrm{p}<.05)$. With the increasing SA, there was also an increase in structural damage of the medial patellar cartilage. In all groups, LTI was significantly lower compared to the control group $(p<.05)$. Furthermore, from grade 2 to grade 4, LTI tended to decrease. Similarly, the MTI of all defect groups was significantly lower than that of the control group $(p<.05)$, and there was a decrease in MTI from grade 2 to grade $4(\mathrm{p}$ $<.05)$. LTI $(\mathrm{r}=-0.46)$ and MTI $(\mathrm{r}=-0.53)$ were moderately correlated with SA.

There was no significant differences in femoral lateral and medial condyle symmetry, trochlear

Table I. - Characteristic of the study population $(n=400)$ (Values are expressed as mean \pm standart deviation) (MPCD : medial patellar cartilage defect)

\begin{tabular}{|l|c|c|c|c|}
\hline & Grade 2 MPCD & Grade 3 MPCD & Grade 4 MPCD & Control group \\
\hline Sample size & 100 & 100 & 100 & 100 \\
\hline Age (year) & $47.3 \pm 5.5$ & $49 \pm 5.7$ & $51.5 \pm 4.9$ & $46.5 \pm 5.2$ \\
\hline Gender (female/male) & $65 / 35$ & $78 / 22$ & $79 / 21$ & $74 / 26$ \\
\hline Right/Left & $45 / 55$ & $50 / 50$ & $56 / 35$ & $57 / 43$ \\
\hline
\end{tabular}


Table II. — Descriptive analyses of trochlear parameters by groups (Values are expressed as mean \pm standart deviation) (MPCD: medial patellar cartilage defect)

\begin{tabular}{|l|c|c|c|c|c|}
\hline & Grade 2 MPCD & Grade 3 MPCD & Grade 4 MPCD & Control group & P value \\
\hline SA & $126.1 \pm 4.5$ & $130.3 \pm 6.2$ & $131.6 \pm 5.7$ & $124.6 \pm 5.8$ & 0.001 \\
\hline LTI & $23.1 \pm 2.2$ & $22.6 \pm 2.5$ & $21.4 \pm 2.7$ & $24.8 \pm 2.5$ & 0.001 \\
\hline MTI & $26.3 \pm 3.2$ & $24.6 \pm 3.3$ & $23.2 \pm 4$ & $29 \pm 2.6$ & 0.001 \\
\hline $\begin{array}{l}\text { Femoral lateral and medial condyle } \\
\text { symmetry }\end{array}$ & $1.01 \pm 0.033$ & $1.02 \pm 0.03$ & $1.03 \pm 0.038$ & $1.03 \pm 0.031$ & 0.17 \\
\hline Trochlear facet asymmetry & $0.68 \pm 0.08$ & $0.65 \pm 0.08$ & $0.66 \pm 0.07$ & $0.67 \pm 0.09$ & 0.79 \\
\hline Trochlear width & $34 \pm 3.9$ & $34.1 \pm 3.9$ & $33.9 \pm 3.7$ & $35 \pm 4$ & 0.08 \\
\hline
\end{tabular}

facet asymmetry, and trochlear width between groups with and without medial patellar cartilge defect $(\mathrm{p}>.05)$

The measurements of trochlear morphology of all groups are summarized in Table II .

\section{DISCUSSION}

One of the main findings of this study was the existence of a flattened medial trochlea in patients with medial patellar cartilage defects. Moreover, it was revealed that compared to the control group, there was a significant decrease in the MTI of patients with medial patellar cartilage defects. Another finding of this study was association between a flattened lateral trochlea and structural damage in the medial patellar cartilage.

Osteoarthritis is a progressive degenerative disease characterized by a gradual loss of articular cartilage (1). Previous findings in the general patellofemoral osteoarthritis population suggest that patellofemoral alignment and trochlear morphology are typically more strongly associated with lateral compartment $(5,6,17)$. However, the association between trochlear morphology and medial patellofemoral osteoarthritis remains unknown.

Numerous studies analyzed the association of trochlear morphology with patellofemoral osteoarthritis. In these studies, SA was used as a measure of trochlear morphology. A flattened and shallow femoral trochlea was characterized by an increased SA. It was also mostly shown that a flattened and shallow trochlea was associated with lateral and medial patellar cartilage structural damage $(2,4,5,7,8)$. However, Weintraub et al. (18) reported that a decreased SA was associated with increased possibility of isolated medial patellofemoral osteoarthritis in young adults. Furthermore, Stefanik et al. (6) demonstrated weak associations between SA and structural damage in the medial patellar cartilage of adults. In contrast, we found a significant correlation between SA and grade of cartilage degeneration. This is in agreement with Tvasalas et al. (7), who demonstrated that a shallow trochlea could lead to patellar instability with subsequent disproportional load distribution across the patellofemoral joint during knee movement. High mechanical stress on the patellofemoral joint can cause structural damage in the articular cartilage.

Research suggests that decreased LTI, representing a flattened lateral trochlea, is associated with lateral patellofemoral osteoarthritis $(5,6)$. To our knowledge, only one study examined the relationship between trochlear morphology and isolated medial patellofemoral osteoarthritis in adults (6). In that study, the authors reported that in patients with medial patellofemoral osteoarthritis who had low LTI had 1.6 times greater risk of cartilage damage compared to those with high LTI. In the present study, we found a significant correlation between the advancing severity of cartilage defect and decreasing LTI. This observation indicates that a flattened lateral trochlea may contribute to the development of cartilage structural damage in the medial patella, which is in agreement with the results of the study by Stefanik et al. (6).

Stefanik et al. (6) reported no association between MTI and medial patellar cartilage defects. In contrast, our patients with medial patellar cartilage defects had a significantly reduced MTI compared to the control group. In addition, a significant correlation with cartilage lesion grading was seen. 
Based on these results, we suggest that a flattened medial trochlea is a risk factor for the development of cartilage structural damage in the medial patella. In full extension, the patella is positioned superior and slightly lateral to the trochlear groove. At the initiation of knee flexion prior to full engagement of the patella with the trochlear groove, patellofemoral alignment remains highly vulnerable to changes in femoral position. Forces that displace the patella further medially during this critical early phase of knee flexion could contribute to medial patellar cartilage damage (6,19-22). Studies analyzing patella malalignment ( medial patellar tilt) with a flattened medial trochlea can help to delineate the pathophysiology of structural damage in the patellar cartilage.

SA is defined as the angle between the medial and lateral trochlear facets (5-7). Stefanik et al (6). reported that LTI was strongly correlated with SA. The authors indicated that a flattened lateral trochlea could contribute to the development of a shallow trochlea. Our study showed that LTI and MTI were moderately correlated with SA. Our results reflect that not only a flattened lateral trochlea but also a flattened medial trochlea might be effective in the development of a flattened and shallow trochlea .

Only few studies in the literature have analyzed the association of trochlear facet asymmetry and trochlear width with patellar cartilage defect $(2,15,16)$. In the present study, we did not find a significant difference in trochlear facet asymmetry and trochlear width between the medial patellar cartilage defect and control groups ; these findings are in agreement with those of previous studies. The femoral lateral and medial condyle symmetry has not been evaluated in previous studies and can be used to assess the trochlear morphology with the LTI and MTI . In our study, no difference was found in femoral lateral and medial condyle symmetry between the medial patellar cartilage defect and control groups. This finding shows that the flattened medial trochlea also plays a role in the flattened and shallow trochlea.

Patellofemoral pain syndrome is a common orthopedic problem that can cause serious disability, and it usually occurs due to chondromalasia patella. Further clinical studies analysing patellofemoral joint geometry with dynamic methods will improve our understanding of the pathophysiology of structural damage in the medial patellar cartilage.Taking the tochlear morphology into consideration in patellofemoral joint evaluation will enable us to design new physical and surgical modalities.

Our study has certain limitations. For example, the study design was retrospective; therefore, it was not possible to evaluate the clinical findings of patients with medial patellar cartilage defects who had undergone an MRI. The diagnosis and grading of the patellar cartilage defects were based on MRI but not correlated to arthroscopic findings. In addition, routine MRI sequences were used for evaluation rather than more specific sequences for the patellar cartilage. Another limitation of our study is that we did not analyse the body mass index of patients.

In conclusion, an abnormal trochlear morphology is associated with structural damage in the medial patellar cartilage. A flattened medial trochlea indicated by MTI is a risk factor for the structural damage of the medial patellofemoral joint cartilage. There is a association between a flattened lateral trochlea and structural damage in the medial patellar cartilage.

\section{REFERENCES}

1. Kim YM, Joo YB. Patellofemoral osteoarthritis. Knee Sur Relat Res 2012 ; 24(4) : 193-200.

2. Mehl J, Feucht MJ, Bode G, Dovi-Akue D, Südkamp NP, Niemeyer P. Association between patellar cartilage defects and patellofemoral geometry : a matched-pair MRI comparison of patients with and without isolated patellar cartilage defects. Knee Surg Sports Traumatol Arthrosc $2014 ; 24: 838-846$.

3. Noehren B, Duncan S, Lattermann C. Radiographic parameters associated with lateral patella degeneration in young patients. Knee Surg Sports Traumatol Arthrosc $2012 ; 20: 2385-2390$.

4. Ali SA, Helmer R, Terk MR. Analysis of the patellofemoral region on MRI : association of abnormal trochlear morphology with severe cartilage defects. AJR Am J Roentgenol. $2010 ; 194$ : 721-727.

5. Duran S, Cavusoglu M, Kocadal O, Sakman B. Association between trochlear morphology and chondromalacia patella: an MRI study. Clinical Imaging 2017 ; $41: 7-10$. 
6. Stefanik JJ, Roemer FW, Zumwalt AC, et al. Association between measures of trochlear morphology and structural features of patellofemoral joint osteoarthritis on MRI : the MOST study. J Orthop Res 2012 ; 30 : 1-8.

7. Tsavalas N, Katonis $\mathbf{P}$, Karantanas AH. Knee joint anterior malalignment and patellofemoral osteoarthritis : an MRI study. Eur Radiol 2012 ; 22 : 418-428.

8. Tuna BK, Semiz-Oysu A, Pekar B, Bukte Y, Hayırlıglu A. The association of patellofemoral joint morphology with chondromalacia patella : a quantitative MRI analysis. Clinical Imaging 2014 ; 38 : 495-498.

9. Harris JD, Brophy RH, Jia G. et al. Sensivity of magnetic resonance imaging for detection patellofemoral articular cartilage defect . Arthroscopy $2012 ; 28$ : 1728-1737.

10. Pihlajamaki HK, Kuikka PI, Leppanen VV,Kiuru MJ, Mattila VM. Reliability of clinical findings and magnetic resonance imaging for the diagnosis of chondromalacia patellae. J Bone Joint Surg Am 2010 ; 92 : 927-934.

11. Recht MP,Piraino DW, Paletta GA, Schils JP, Belhobek GH. Accuracy of fat-suppressed three -dimensional spoiled gradient-echo FLASH MR imaging in the detection of patellofemoral articular cartilage abnormalities. Radiology 1996 ; $198: 209-212$.

12. Chhabra A, Subhawong TK, Carrino JA. A systematised MRI approach to evaluating the patellofemoral joint. Skeletal Radiol $2011 ; 40$ : 375-378.

13. Paiva M, Bland L, Hölmich P. et al. Quality assessment of radiological measurements of trochlear dysplasia; a literature review. Knee Surg Sports Traumatol Arthrosc $2018 ; 26: 746-755$.

14. Diederichs G, Issever AS,Scheffler S. MR imaging of patellar instability : Injury patterns and assessment of risk factors. RadioGraphics $2010 ; 30$ : 961-981.
15. Sebro R, Weintraub $S$. Knee morphometric and aligment measurements with MR imaging in young adults with central cartilage lesions of the patella and trochlea. Diagn Interv Imaging 2017 ; 98 : 429-40 .

16. Sebro R, Weintraub S. Association between lateral patellar osteoarthrosis and knee morphology and aligment in young adults. Clin Radiol 2017 ; 72 : 793.e11-793.e18.

17. Macri EM, Felson DT, Zhang Y. et al. Patellofemoral morphology and aligment : refence values and doseresponse patterns for the relation to MRI features of patellofemoral osteoarthritis. Osteoarthritis Cartil 2017; 25 : 1690-1697.

18. Weintraub S, Sebro R. Superolateral hoffa's fat pad edema and trochlear sulcal angle are associated with isolated medial patellofemoral compartment osteoarthritis. Can Assoc Radiol J 2018 ; 69 : 450-457.

19. Besier TF, Garry EG, Scott LD, Fredericson M, Beaupre GS. The influence of femoral internal and external rotation on cartilage stresses within the patellofemoral joint. $J$ Orthop Res. 2008 ; 26:1627-1635.

20. Gross KD, Felson DT, Niu J. et al. Association of flat feet with knee pain and cartilage damage in older adults. Arthritis Care Res ( Hoboken ) 2011 ; 63:937-944.

21. Gross KD, Niu J, Stefanik JJ. et al. Breaking the law of valgus : the suprising and unexplained prevalence of medial patellofemoral cartilage damage. Ann Rheum Dis 2012 ; 71:827-1832.

22. Hefzy MS, Jacson WT, Saddemi SR,Hsieh YF. Effects of tibial rotations on patellar tracking and patello-femoral contact areas. J Biomed Eng 1992 ; 14: 329-343. 